\title{
Germanica
}

\section{L'« autre » beauté de Joséphine Baker}

Die « andere » Schönheit der Josephine Baker

\section{Pascale Avenel-Cohen}

\section{(2) OpenEdition}

Journals

Édition électronique

URL : http://journals.openedition.org/germanica/449

DOI : 10.4000/germanica.449

ISSN : 2107-0784

Éditeur

Université de Lille

\section{Édition imprimée}

Date de publication : 1 décembre 2005

Pagination : 69-78

ISBN : 2-913857-16-7

ISSN : 0984-2632

Référence électronique

Pascale Avenel-Cohen, «L'« autre » beauté de Joséphine Baker », Germanica [En ligne], 37 | 2005, mis en ligne le 07 janvier 2010, consulté le 06 octobre 2020. URL : http://journals.openedition.org/ germanica/449; DOI : https://doi.org/10.4000/germanica.449

Ce document a été généré automatiquement le 6 octobre 2020.

(c) Tous droits réservés 


\title{
L'« autre » beauté de Joséphine Baker
}

Die « andere » Schönheit der Josephine Baker

\author{
Pascale Avenel-Cohen
}

1 "Cet été, elle a suscité l'envie les "dames mondaines" par la majesté de sa démarche légère ». "Brillante comme le bronze, jeune, coquette ${ }^{1}$ ", voici, non pas Joséphine Baker, mais Kadidja, la fille du chef africain Hersy Egga, l'un des « visiteurs » réguliers du parc animalier de Carl Hagenbeck à Stellingen aux environs de Hambourg, ou plutôt l'un de ses hôtes, car il contribuait à la couleur locale africaine du parc avec d'autres habitants de son village. La description de sa fille s'impose tout naturellement en 1928 dans la mesure où, après leur séjour à Stellingen au printemps, les membres de son clan venus en Allemagne cette année-là, se retrouvent exposés au zoo de Berlin dans une reconstitution de leur village. Mais aussi belle soit cette jeune fille de dix-sept ans, le rédacteur de l'article n'en oublie pas moins de spécifier qu'elle ne sera jamais qu'une curiosité exotique parmi d'autres et qu'à ce titre sa beauté, est, elle aussi, purement exotique. D'ailleurs, cette coexistence avec des Européens standard ne peut être qu'éphémère ${ }^{2}$. Avant d'être caractérisée par sa beauté, cette jeune femme l'est donc par son altérité, à moins que le barrage de l'altérité ne jaillisse de sa beauté même, tel un garde fou au secours d'Européens déstabilisés par un retour en force de leur sensualité.

2 À en croire Paul Eipper, l'auteur de l'article sur Kadidja, les femmes seraient les premières à se précipiter pour visiter le zoo. Elles sont d'ailleurs régulièrement accusées de voyeurisme et de lubricité ${ }^{3}$, à croire que les hommes allemands seraient restés de marbre, à moins qu'ils n'aient satisfait leurs fantasmes dans les colonies ${ }^{4}$. Mais, la grande majorité des textes publiés sur Joséphine Baker ou les rares «beautés noires » de passage en Allemagne sont rédigés par des hommes, qui n'ont de cesse de souligner la distance qui peut exister entre ces femmes, aussi superbes qu'animales, et l'homme civilisé qu'ils incarnent. Ainsi, en même temps qu'il affirme une prétendue supériorité de Kadidja sur les mondaines berlinoises, Paul Eipper justifie celle-ci par le port altier de la jeune femme, par cette même démarche de panthère que l'on associe à Joséphine Baker, une démarche souple et aérienne, certes, mais non humaine. Qu'elles 
soient considérées comme une "jolie gazelle $»^{5}$ ou une féline, il n'est jamais fait référence à leurs traits et, dans les premières années, ce ne sont pas les formes de Joséphine Baker qui retinrent l'attention de la presse, mais sa stupéfiante souplesse et ses grimaces ${ }^{6}$.

Or cette supériorité physique indéniable se retourne contre elle, car elle est implicitement synonyme d'absence d'esprit, et ce quelle que soit l'approche de la rationalité que l'on choisisse. Norbert Elias montre ainsi dans La Dynamique de l'occident ${ }^{7}$, que "l'autocontrainte» et la pudeur par laquelle les humains, et en particulier les Occidentaux, ont étouffé leurs sentiments au fil des siècles, au fur et à mesure qu'ils montaient dans la hiérarchie sociale, est ensuite devenue une sorte de code de l'honneur assimilé à la « civilisation ». Dans sa perspective, la rationalité n'est qu'un corollaire de l'autocontrainte, à une époque où l'état féodal avait atteint une certaine taille et que la Raison devenait essentielle à sa cohérence pour éviter des actes de vaillance, certes très nobles, mais parfois désastreux. Toutefois, son avènement aurait approfondi le gouffre entre les hommes «civilisés » et les autres, incapables de se contrôler suffisamment pour faire passer les impératifs de l'esprit avant les élans spontanés. Bien qu'il ne partage pas cette vision utilitariste de la raison, Léon Poliakov arrive aux même conclusions quant à l'exclusion d'une bonne part de l'humanité au nom de la rationalité. Dans ce cas, non seulement la maîtrise, mais surtout l'affirmation de prévalence absolue de l'esprit sur le corps et donc l'exclusion de toute sensualité dans les relations sociales, constitue le critère principal de définition de l'humain. Or, pour citer Poliakov, «la rigueur des condamnations permet d'inférer la force des tentations, la répugnance culturelle atteste la sévérité d'un tabou qui ne pouvait que stimuler l'attirance biosexuelle contrariée 8 . " Ainsi, l'altérité constituée comme garde fou pour prouver "scientifiquement" la supériorité blanche et empêcher toute transgression raciale est-elle une arme à double tranchant, car aussi rationnel soit-il, l'homme civilisé est sujet à la tentation.

4 Le bannissement de toute sensualité des relations sociales a d'ailleurs toujours été très relatif. Il ne signifie en rien que la pornographie n'ait pas existé, mais à partir d'un certain niveau social, elle respectait une codification précise et n'avait pas sa place dans les échanges officiels, à moins qu'ils n'aient été sciemment subversifs. Habitués depuis longtemps à côtoyer le monde de la prostitution quand le besoin s'en faisait sentir, les Allemands de la République de Weimar, comme bon nombre des autres Européens, n'y voyaient aucune remise en question de la domination européenne sur le reste du monde. Cela participait de la césure entre vie publique et vie privée qui permettait au système social de rester supportable et cantonnait la sexualité dans des zones bien définies dont tout le monde avait complaisamment fini par s'accommoder.

5 L'arrivée de Joséphine Baker perturbe cette routine non pas tant par la sensualité dont elle est censée faire étalage que par les réactions exacerbées qu'elle provoque chez les spectateurs en réveillant publiquement leur propre sensualité refoulée. La critique virulente de bien des détracteurs de l'artiste relève en général d'une peur primaire. Ils imaginent soudain, en voyant disparaître les derniers remparts qui séparaient l'homme "civilisé ", ataraxique en théorie, du "sauvage », lubrique, que la "Revue Nègre臨》 menace la civilisation elle-même. En effet, si l'homme civilisé devient lui aussi lubrique, plus rien ne le distingue alors du sauvage. Dans un tel contexte, il devient psychologiquement fondamental d'ériger des barrières entre la femme qui a réveillé publiquement ces instincts réprimés et les détenteurs de la civilisation. Rabaisser la 
femme noire au rang animal participe de cette stratégie. Si ce discours animalisant ne suffit pas éliminer le désir, il vise tout au moins à transformer le passage à l'acte en tabou et à protéger in extremis la supériorité des Blancs.

6 L'arsenal utilisé à cet égard est bien éprouvé : mis au point lors de la conquête du Nouveau Monde par les Espagnols, il n'a presque pas évolué en cinq siècles. Il est d'ailleurs assez saisissant de constater à quel point ces poncifs ont résisté au temps. Bien qu'ils ne s'adressent pas à la même population, puisqu'ils sont antérieurs à la traite des esclaves africains, les motifs sont strictement les mêmes : il s'agit d'affirmer une altérité radicale qui exclut la moindre ressemblance entre l'homme civilisé quel qu'il soit, et tout Autre. Rien n'a été ajouté depuis le texte de Jean de Léry, Histoire d'un voyage faict en la terre du Brésil, publié en $1578^{9}$. Si, dans son analyse, Michel de Certeau met sur le même plan Sauvage et Civilisé, il n'en est pas de même pour Léry ou pour tous ses épigones des siècles postérieurs : il est tout à fait naturel aux Occidentaux colonisateurs de considérer les autres comme une catégorie indifférenciée et monolithique ce qui justifie le singulier dans les expressions «le Nègre», «le sauvage », « le primitif». Cela va nettement moins de soi s'agissant des Blancs, toujours au pluriel. Passant outre ce déséquilibre manifeste, l'historien élabore à partir de ce récit archétypal un schéma d'opposition entre le "Sauvage » et le "Civilisé » tout à fait applicable au discours sur Joséphine Baker dans l'Allemagne des années vingt :

Bien sûr, le sauvage est nu. Soit dit en passant, Joséphine Baker, élevée dans une Amérique puritaine, n'a acceptée de danser quasiment nue qu'à la demande des cabarets européens ${ }^{10}$.

7 Il ne porte pas de vêtement mais un ornement, généralement à base de plumes. C'est bien dans cet esprit que fut conçue pour la danseuse américaine la fameuse ceinture de plumes d'abord, puis de bananes dès 1926.

8 Le sauvage est oisif, il ne travaille pas, mais festoie constamment. Dans ce domaine, les contemporains honnêtes reconnaissaient certes aux danseurs et jazzmen noirs une véritable préparation, mais qui n'enlevait rien au caractère festif de leur activité.

9 La notion de cohésion du groupe ne peut pas s'appliquer ici, dans la mesure où Joséphine Baker a très vite volé de ses propres ailes après la tournée de la Revue Nègre avec laquelle elle était venue en Europe pour la première fois en 1925 à l'âge de dixneuf ans. En outre, la parfaite synchronisation des troupes de girls américaines, qui frappa tant les esprits en Allemagne, n'était pas l'apanage des revues noires.

La dernière des cinq catégories, selon laquelle le Sauvage s'adonne au plaisir tandis que le Civilisé se plie a l'éthique est, quant à elle, reprise sans la moindre hésitation puisque sa sensualité débridée est l'un des caractères les plus volontiers prêtés à Joséphine Baker et à ses représentations littéraires.

11 En outre, l'affirmation d'une bonne santé éhontée et voyante affirmée par Jean Léry ${ }^{11}$, alors que la majeure partie des populations autochtones succombèrent aux maladies que leur transmirent les Espagnols ou furent tout bonnement massacrés dans les quatre décennies qui suivirent la conquête, participe de l'altérité radicale des AfroAméricaines sous la République de Weimar.

12 Le personnage de Becky Floyd, allusion transparente à Joséphine Baker dans la nouvelle de Bruno Frank intitulée Politische Novelle en 1928 est particulièrement caractéristique de cette vision fantasmée extrême de la femme noire, sinon de Joséphine Baker, ellemême élevée au rang d'archétype ${ }^{12}$. Bien que dans son article Marc Weiner insiste surtout sur les prétendus effets pernicieux du jazz comme incitateur à la débauche ${ }^{13}$, il 
n'en reste pas moins que le personnage féminin déborde d'une sensualité irrépressible et dangereuse indépendamment de la musique. Bien qu'elle n'apparaissent que dans douze pages sur cent vingt cinq, mis à part le souvenir nostalgique final du protagoniste qui succombe d'ailleurs assassiné dans les bras d'une prostituée malgache, donc "noire ", le personnage de la danseuse est particulièrement saisissant. Même si elle est présentée comme intelligente puisqu'elle interrompt sa prestation dès qu'elle sent fléchir l'attention du public, distraite par autre chose, elle se définit avant tout par sa sensualité tout droit venue de la « jungle $»^{14}$ («Urwald»). Au demeurant il est clair que cette "impératrice africaine " ("afrikanische Kaiserin» p.107) est américaine comme le prouve son nom, mais un tel détail ne change rien au fantasme de la femme noire, nécessairement animalisée. Ses " yeux » pour en être " magnifiques » n'en sont pas moins "animaux» («ihre herrlichen Tieraugen» p.47) et elle a une endurance exceptionnelle qui fait qu'à quatre heures du matin, après une longue journée de travail, « son corps était aussi frais que seize heures auparavant» («Wenn sie dort um die vierte Morgenstunde schied, so war ihr Körper so frisch wie -sechzehn Stunden zuvor ", p. 40).

13 Le personnage de Becky Floyd est passionnant dans la mesure où, peut-être plus encore que les articles haineux sur Joséphine Baker qui se résument généralement à quelques insultes racistes particulièrement virulentes mais d'une banalité consternante, il est révélateur du fantasme que suscita l'artiste et auquel les commentateurs réagirent bien plus qu'à sa prestation réelle. C'est ainsi que Bruno Frank fait effectuer à son personnage un strip-tease d'une grande agressivité :

Mais elle montait maintenant sa chaste robe de plus en plus haut, on voyait maintenant ses genoux, maintenant ses cuisses, elle tournoyait portée par l'ivresse de ses forêts [vierges] et dans un cri perçant, aigu, clair comme le cristal, elle arracha des deux poings en un seul geste la robe et le bustier, ses seins étrangers, pointus, les pointes teintes de pourpre, jaillirent dans la lumière et, entièrement nue, seules ses parties honteuses tout juste couvertes, elle s'exposait en tournant dans un formidable délire grotesque ${ }^{15}$.

14 On le remarque immédiatement, outre une animalité exacerbée, renforcée par les allusions à la forêt vierge et à un cri, lui aussi à sa place dans la jungle, les seins de Becky Floyd sont « étrangers» («fremd»). Or, il s'agit là d'un attribut essentiel de la femme et de l'érotisme. Ceci prouve à quel point il importait aux Allemands de cette époque de ne pas mettre sur le même plan l'érotisme blanc et l'érotisme noir. Joséphine Baker aussi bien que son fantasme sous la forme de Becky Floyd n'étaient pas présentées comme de très belles femmes dont la nudité excitait les spectateurs, mais comme une beauté autre, étrangère, animale et pernicieuse.

Sous la forme fantasmée de Becky Floyd, l'artiste ne fait pas mystère de ses intentions cannibales. Avant de terminer son spectacle en apothéose, elle invite tous les jours sur scène un spectateur avec lequel elle se livre à un jeu sado-masochiste en le faisant danser avec elle. Mais ce n'est pas tant la lubricité et la soumission volontaire de la victime exposée au grand jour que tient à dénoncer l'auteur que la dimension éminemment vorace de la danseuse dont la bouche se transforme pour l'occasion en un " entonnoir africain de chair, qu'il a toujours été » "dans un rictus sournois » ${ }^{16}$. Car cette beauté "étrangère ", irrésistible, ensorcelante, est avant tout destructrice. Or, l'association faite ici entre la couleur noire et les mangeurs d'hommes ne relève n'est pas seulement d'une assimilation rapide entre la couleur foncée de peau et le cannibalisme, mais aussi du topos de la vagina dentata, du sexe denté, de la voracité 
féminine tueuse d'homme. Cette représentation de la femme n'est pas nouvelle, au fil du temps elle est devenu un lieu commun de la littérature, mais associée à Joséphine Baker, le motif est réactivé dans la mesure où il superpose plusieurs strates de la représentation cannibale: celle de la femme, celle du "primitif» nécessairement mangeur d'homme blanc, celle d'un monde sauvage incarné par la féminité ${ }^{17}$, mais aussi celle d'une Amérique écrasant l'Allemagne vaincue, non seulement par son économie triomphante, mais aussi par sa culture de masse.

Car ce qui effraie le plus les commentateurs, c'est le succès triomphal de Joséphine Baker qu'ils interprètent comme celui du nouveau monde sur l'ancien dans une perspective vitaliste superficielle. Ses plus ardents partisans ne sont d'ailleurs pas loin de penser la même chose, si ce n'est que, plutôt qu'une destruction, ils y voient la régénération de la culture allemande par un sang neuf et une culture en devenir. Or, ces deux interprétations découlent du même mode de pensée initial : celui du rejet de l'autre et de la constitution d'une altérité radicale dont le contact avec l'Occident ne peut s'effectuer que de manière brutale, quel qu'en soit le résultat. La subversion de Joséphine Baker vient d'une altérité qui dépasse de loin le simple exotisme.

Cette réception de la «Revue Nègre海》" et de la suite de la carrière de cette artiste semble d'autant plus singulière aujourd'hui que la Revue qui fit le tour de l'Europe était un produit de music-hall fabriqué sur mesure et réalisé par des artistes américains nés pour la plupart dans des grandes villes, comme Joséphine Baker elle-même. Les Allemands ne sont d'ailleurs pas les seuls à en faire un parangon du Sauvage puisqu'en 1931 les organisateurs français de l'exposition coloniale envisagèrent un temps de lui faire incarner la « reine des colonies $»^{18}$.

18 Il est certain que les Européens, et en particuliers les hommes, ne sont pas seuls responsables de cette altérité fabriquée de toutes pièces. En tant que femme de spectacle, Joséphine Baker s'est prêtée au jeu de l'exotisme. Intelligente, elle n'a pas hésité à se promener avec un léopard ${ }^{19}$ ce qui nourrit le fantasme d'animalité de ses spectateurs. Elle n'a rien fait dans un premier temps qui puisse contredire l'image que l'on plaquait sur elle et qui lui apportait le succès. Elle en avait d'ailleurs l'habitude après avoir débuté comme Chorus-girl clownesque dans la bonne vieille tradition raciste américaine ${ }^{20}$, à une époque où la ségrégation était encore loin d'être ébranlée. Elle n'avait d'ailleurs pas d'autre choix si elle voulait échapper à des conditions de vie plus que médiocres. Ses grimaces si remarquées dans la «Revue Nègre医》, pourtant typiques de cette tradition américaine de clownerie attribuée aux Noirs, n'ont d'ailleurs en rien parasité la construction de l'image de sauvage africaine de la part des spectateurs allemands ou français. Elle s'intégrait dans le poncif du primitif festif perpétuellement puéril, toujours en train de s'adonner à la fête. Que les siècles d'esclavage et les millions de morts qui suivirent l'établissement de ces schémas de pensée n'aient pas réussi à mettre en pièce ces images d'Épinal en dit long sur le travail d'aveuglement des Occidentaux. Dans de telles conditions, la question qui se pose est toute autre : Si Joséphine Baker renvoie aux Allemands l'image de la femme noire qu'ils ont en tête sous une forme parfaite, puisque c'est une caricature fabriquée sur mesure, comment se fait-il qu'ils en aient été si surpris et si offusqués? En quoi la prestation de Joséphine Baker posait-elle un problème nouveau?

19 Il est vraisemblable que la difficulté vienne paradoxalement du fait que Joséphine Baker était américaine et non africaine. Cette nationalité ajoute en effet une strate interprétative supplémentaire. À la peur d'une africanisation de l'Allemagne, gagnée 
par l'art nègre et les tentatives expressionnistes de retour à l'homme noir originel, s'ajoute la peur de l'américanisation, le tout dans une même perspective vitaliste simpliste d'opposition entre des cultures jeunes, africaine (sic) et anglo-saxonne d'une part, occidentale ou allemande d'autre part.

Car, si tous se délectaient d'une image d'Africaine entièrement fabriquée, même si un grand nombre finissait par y croire, tous connaissaient parfaitement la nationalité de Joséphine Baker et le contexte dans lequel elle était arrivé sur le vieux continent, à savoir le déferlement du jazz sur l'Europe et sur une Allemagne vaincue qui n'y était absolument pas préparée, culturellement coupée des États-Unis depuis des années. Le succès retentissant de l'artiste et la haine qu'elle a pu susciter sont donc consécutifs à deux phénomènes antinomiques dans le principe mais parfaitement concomitants : la fragilisation des Allemands en tant que détenteurs de la civilisation occidentale, donc dépourvue de désir, rappelés à leur propre sensualité par le contact direct avec une pseudo "sauvage » et le sentiment de disparition de leur culture, intellectuelle et raffinée, écrasée par la société de consommation américaine et ses productions de masse. Bien qu'il semble aujourd'hui difficile de tenir ces deux discours à la fois, cela ne posait alors aucune difficulté particulière. C'est même l'un des traits caractéristiques de cette époque.

21 Mais les détracteurs de Joséphine Baker ne sont pas les seuls à lui dénier un érotisme à l'Européenne. Ses plus fervents admirateurs s'évertuent eux aussi à lui conférer une autre beauté, une beauté androgyne cette fois, que peuvent aussi lui reprocher certains de ses ennemis. Bien que l'androgynie relève là-aussi du mythe et renvoie aux divinités africaines ${ }^{21}$, elle relève cette fois-ci de l'argument de la modernité qui oppose le corps féminin européen, tout en rondeur, au corps d'adolescentes sportives des Américaines ${ }^{22}$. Dans cette perspective, on l'aura compris, c'est le caractère américain de Joséphine Baker que les commentateurs mettent en avant. Cette féminité autre, car androgyne, est alors celle du renouveau, celle de la culture physique, de l'efficacité pragmatique, de la renaissance d'un corps enfin débarrassé de ses connotations négatives. Ce corps si souple et si obéissant n'est pas un boulet, c'est un outil. L'androgynie n'est d'ailleurs pas l'apanage des artistes noires, c'est l'une des caractéristiques essentielles des troupes de girls américaines blanches comme les Tiller-girls ou les Hoffmann-girls de passage en Allemagne en 1923. En tant qu'androgyne, que beauté non allemande, Joséphine Baker rejoint les garçonnes, un autre type de femmes, les sportives desérotisées, sans toutefois jamais se confondre avec elles, car sa couleur n'est jamais oubliée.

Ce sont tous ces niveaux d'interprétation qui, associés, contribuent à constituer l'altérité radicale de Joséphine Baker et de sa beauté, altérité ressentie ensuite comme une menace ; pourtant, si l'artiste n'avait été qu'une beauté exotique parmi d'autres, à l'instar de celles exhibées dans les zoos et sur les champs de foire comme, en son temps, la Vénus hottentote ${ }^{23}$, elle n'aurait vraisemblablement pas été perçue comme un danger. Mais elle chantait et dansait dans des spectacles parfaitement bien huilés, efficaces et relevant d'une rigueur mécanique. En outre, elle n'était plus une rareté exotique, car les jazzmen noirs investissaient l'Europe. Il ne fait pas de doute que c'est là un facteur clé qui explique la panique de certains: Joséphine Baker était triomphante. Mais il n'est pas certain que la repousser dans une altérité radicale ait été la bonne solution pour rendre sa beauté inoffensive. Au contraire, l'animalisation qui 
visait à protéger la supériorité blanche, puis la déféminisation sur le modèle américain, se retournèrent contre les Européens dans la perspective vitalist.

\section{NOTES}

1. Paul Eipper : « Kadidja, Rotflügel und andere Exoten », in Der Querschnitt, 1928, H. 9, p. 631-636 : «bronzeglänzend, jung, kokett [...] und in diesem Sommer die Berliner 'mondänen Damen' mit Neid erfüllt » p. 632.

2. «Und wie sind sie jetzt, jene exotische Schönheiten, die für kurze Zeit ihre Spuren neben der meinen in den Sand gezeichnet haben?», id., p. 631.

3. Cf. Par exemple les plaisanteries de mauvais goût dans "Geschichten von Negern ", in Das Tagebuch, 1929, H. 27, p. 1130.

4. Lilli Jannasch, Schwarze Schmach und schwarz-weiß-rote Schande, 1921, Berlin, Verlag Neues Vaterland, Flugschriften des Bundes Neues Deutschland, Nr.18/21, p. 10.

5. Peter Scher, « Josephine in München », in Das Tagebuch, 1929, H.8, p. 323-324.

6. Ottomar Starke, «Revue Nègre. Anläßlich des Berliner Gastspiels der Negertruppe », in Der Querschnitt, 1926, H. 2, p. 118-120.

7. Traduction de Pierre Kamnitzer du tome 2 de Über den Prozess der Zivilisation ( $1^{\text {re }}$ ed 1939, 2 d ed 1969), Calmann-Lévy, Paris 1991.

8. Léon Poliakov, Le Mythe aryen, Pocket agora, Paris, 1994, p. 166.

9. Michel de Certeau, L'Écriture de l'histoire, NRF Gallimard 1975, p. 237-238.

10. Id., p. 28. Il n'est peut-être pas inutile à ce propos de rappeler que $90 \%$ de la population d'Amérique du Sud et d'Amérique centrale a été décimée dès les premières décennies suivant la conquête.

11. Michel de Certeau, p. 244.

12. L'édition utilisée ici est celle de Reclam, Stuttgart, 1968.

13. Marc A. Weiner : « Urwaldmusik and the Boders of German Identity : Jazz in Literature of the Weimar Republic », in German Quaterly, 1991, n²4, p. 475-487.

14. Bruno Frank, p. $38,44,47$.

15. «Sie aber raffte ihr sittiges Kleid nun höher und höher, jetzt sah man die Knie, jetzt die Schenkel, sie kreiste im Rausch ihrer Wälder, und unter einem durchdringenden hohen glashellen Schrei riß sie sich mit ihren beiden Fäusten zugleich Rock und Mieder vom Leibe, ihre fremden Brüste, spitz, die Knospen purpurn gefärbt, sprangen ins Licht, und völlig nackt, die Scham nur eben bedeckt, gab sie sich drehend preis in gewaltig groteskem Rasen. », p. 44.

16. «Becky Floyds Mund, der eben noch so töchterlich gelächelt, wölbte sich plötzlich zu dem afrikanischen Fleichtrichter, der er eigentlich war, mit einem kleinen tü-ckischen Grinsen blickte sie sich im Saale um und ersah sich einen beleibte, glatzköpf-igen Herrn, der friedlich bei seiner Gattin saß. ", p. 42.

17. Il suffit pour s'en convaincre de regarder l'une des nombreuses représentations de l'Occident découvrant l'Amérique dont la gravure de Jan van der Straet pour l'Americae decima pars de JeanThéodore de Bry, Oppenheim, 1619 : «L'explorateur (A. Vespucci) devant l'Indienne qui s'appelle Amérique ", dans Michel de Certeau, L'écriture de l'histoire, p. 1. 
18. Gérard Le Coat : «Art nègre, revue négre : Esthétique primitiviste et syndrome raciste en France (1905-1935)», in Antoine Régis (ed) : Carrefour de Cultures. Mélanges offerts à Jacqueline Leiner, Tübingen, Nazz, 1993, p. 23-34, ici p. 33.

19. Wendy Martin: "'Remembering the Jungle' : Josephine Baker and Modernist Parody ", in Barkan Elazar (ed), Bush Ronald (ed), Prehistories of the Future: The Primitivist Project and the Culture of Modernism, Standford United Press, 1995, p. 310-325, ici p. 322.

20. Karen C.C. Dalton, Henry-Louis Jr Gates, « Josephine Baker and Paul Colin : African Dance seen through Parisian Eyes », in Critical Unquiry, 1998, Summer 24 (4), p. 903-34, ici 909-911.

21. Joachim Schultz, Wild, irre und rein: Wörterbuch zum Primitivismus der literarischen Avantgarde in Deutschland und Frankreich zwischen 1900 und 1940, Anabas, Gießen Verlag, 1995, p. 29 et 33-34.

22. Cf. Fritz Giese, Girlkultur. Vergleiche zwischen amerikanischem und europäischem Rhythmus und Lebensgefühl, Munich, Delphin, 1925.

23. Elle avait été ramenée en Grande-Bretagne par un chirurgien britannique et fut exhibée vers 1810. Cuvier en fit des moulages qui furent longtemps exposés à Paris au Musée de l'Homme.

\section{RÉSUMÉS}

En dépit de son immense succès en Allemagne dans la Revue Nègre et du pouvoir de séduction qui lui était unanimement reconnu, Joséphine Baker ne fut jamais louée directement pour sa beauté sans qu'il soit spécifié que cette beauté n'était pas de même nature que celle des Blanches. Les commentateurs élaborèrent pour elle une altérité radicale, constituée à partir de lieux communs et de schémas de pensée bien antérieurs. Se mêlaient et se superposaient alors des figures de l'altérité diverses et parfois contradictoires : celle de la féminité, de la primitivité, de la modernité américaine, de l'androgynie... Le but de ces stratégies de différenciation n'était autre que de désamorcer tout danger de dénaturation de l'identité allemande en affirmant une altérité insurpassable, à moins qu'il ne s'agisse de mettre en valeur la vitalité d'une beauté primitive qui aurait pu régénérer une culture allemande raffinée mais exsangue. Mais, en constituant un fantasme très fort, tous conférèrent à la figure de Joséphine Baker un formidable pouvoir de subversion qui se retourna contre ceux que son altérité était censée protéger.

Trotz ihres enormen Erfolges in Deutschland im Rahmen der Neger Revue und einer Verführungsmacht, die ihr keiner bezweifelte, wurde Josephine Baker nie direkt für ihre Schönheit belobt, ohne dass das Fremde an ihr im Vergleich mit den Weißen unterstrichen wurde. Für sie erarbeiteten die Kritiker eine radikale Fremdheit aus einer Mischung von viel älteren Gemeinplätzen und Denkarten. So verschmolzen verschiedene manchmal sogar gegensätzliche Figuren der Fremdheit: Weiblichkeit, Primitivität, amerikanische Modernität, Doppelgeschlechtigkeit... Das Ziel dieser Strategien der Abtrennung war es, einerseits jegliche Gefahr einer «Entartung » der deutschen Identität zu vermeiden, indem das Fremde dieser Schönheit als unüberwindbar behauptet wurde, oder andererseits die Lebenskraft dieser primitiven Schönheit $\mathrm{zu}$ betonen, die eine überfeine aber kraftlose deutsche Kultur hätte regenerieren können. Aber, indem sie eine so prägnante Vorstellung schufen, vergaben alle der Figur der Josephine Baker eine gewaltige Kraft der Subversion, die eben jene bedrohte, die jene Fremdheit schützen sollte. 
INDEX

Mots-clés : beauté, laideur

\section{AUTEUR}

\section{PASCALE AVENEL-COHEN}

Université Charles-de-Gaulle - Lille 3 